\title{
Effectiveness of initiating extrafine-particle versus fine-particle inhaled corticosteroids as asthma therapy in the Netherlands
}

Thys van der Molen ${ }^{1}$, Dirkje S. Postma ${ }^{2}$, Richard J. Martin ${ }^{3}$, Ron M. C. Herings ${ }^{4}$, Jetty A. Overbeek ${ }^{4}$, Victoria Thomas ${ }^{5}$, Cristiana Miglio ${ }^{5}$, Richard Dekhuijzen ${ }^{6}$, Nicolas Roche ${ }^{7}$, Theresa Guilbert ${ }^{8}$, Elliot Israel ${ }^{9}$, Wim van Aalderen ${ }^{10}$, Elizabeth V. Hillyer ${ }^{5}$, Simon van Rysewyk ${ }^{11}$ and David B. Price ${ }^{11,12^{*}}$

\begin{abstract}
Background: Most randomised clinical trials typically exclude a significant proportion of asthma patients, including those at higher risk of adverse events, with comorbidities, obesity, poor inhaler technique and adherence, or smokers. However, these patients might differentially benefit from extrafine-particle inhaled corticosteroids (ICS). This matched cohort, database study, compared the effectiveness of extrafine-particle with fine-particle ICS in a reallife population initiating ICS therapy in the Netherlands.

Methods: Data were from the PHARMO Database Network, comprising pharmacy and hospital discharge records, representative of $20 \%$ of the Dutch population. The study population included patients aged $12-60$, with a General Practice-recorded diagnosis for asthma (International Classification of Primary Care code R96), when available, $\geq 2$ prescriptions for asthma therapy at any time in their recorded history, and receiving first prescription of ICS therapy as either extrafine-particle (ciclesonide or hydrofluoroalkane beclomethasone dipropionate [BDP]) or fine-particle ICS (fluticasone propionate or non-extrafine-particle-BDP). Patients were matched (1:1) on relevant demographic and clinical characteristics over 1-year baseline. Primary outcomes were severe exacerbation rates, risk domain asthma control and overall asthma control during the year following first ICS prescription. Secondary outcomes, treatment stability and being prescribed higher versus lower category of short-acting $\beta 2$ agonists (SABA) dose, were compared over a 1-year outcome period using conditional logistic regression models.
\end{abstract}

Results: Following matching, 1399 patients were selected in each treatment cohort (median age: 43 years; males: $34 \%$ ). Median (interquartile range) initial ICS doses (fluticasone-equivalents in $\mu \mathrm{g})$ were $160(160-320)$ for extrafine-particle versus 500 (250 - 500) for fine-particle ICS ( $p<0.001)$. Following adjustment for residual confounders, matched patients prescribed extrafine-particle ICS had significantly lower rates of exacerbations (adjusted rate ratio [95 \% Cl], 0.59 [0.47-0.73]), and significantly higher odds of achieving asthma control and treatment stability in the year following initiation than those prescribed fine-particle ICS, and this occurred at lower prescribed doses. Patients prescribed extrafine-particle ICS had lower odds of being prescribed higher doses of SABA (0.50 [0.44-0.57]).

Conclusion: In this historical, matched study, extrafine-particle ICS was associated with better odds of asthma control than fine-particle ICS in patients prescribed their first ICS therapy in the Netherlands. Of importance, this was reached at significantly lower prescribed dose.

Keywords: Asthma, Effectiveness, Extrafine-particle, Fine-particle, Inhaled corticosteroids

\footnotetext{
* Correspondence: dprice@rirl.org

${ }^{11}$ Observational \& Pragmatic Research Institute Pte, Ltd, Singapore, Singapore

${ }^{12}$ Academic Primary Care, University of Aberdeen, Polwarth Building, Foresterhill, AB25 2ZD, Aberdeen, UK

Full list of author information is available at the end of the article
} International License (http://creativecommons.org/licenses/by/4.0/), which permits unrestricted use, distribution, and reproduction in any medium, provided you give appropriate credit to the original author(s) and the source, provide a link to the Creative Commons license, and indicate if changes were made. The Creative Commons Public Domain Dedication waiver (http://creativecommons.org/publicdomain/zero/1.0/) applies to the data made available in this article, unless otherwise stated. 


\section{Background}

Traditional randomized controlled trials (RCT) aim to establish a clear cause-and-effect relationship between an intervention and an outcome. Although asthma RCTs have high internal validity, a problem is that they typically represent fewer than $5 \%$ of patients with a current diagnosis of asthma [1, 2]. Patients at high risk of adverse events, with comorbid conditions such as obesity, rhinitis or smoking, or those with poor inhaler technique and adherence, are typically excluded. Real-life research can help balance the limitations of a RCT design [1]. Using real-life medical information recorded in primary care databases allows assessment of long-term outcomes in broader asthma populations cared for under usual conditions. These populations also include patients with unstable asthma often excluded from RCTs, but routinely seen by clinicians in primary care settings [3].

Traditional inhaler devices for controlling asthma symptoms produce fine particles with a mass median aerodynamic diameter (MMAD) of $2-4 \mu \mathrm{m}$; however, some newer pressurized metered dose inhalers (pMDI) using hydrofluoroalkane propellant generate an aerosol of smaller, extrafine-particles with an MMAD $<2 \mu \mathrm{m}$ $[4,5]$. RCTs $8-12$ weeks in duration have reported that extrafine-particle ICS is equally effective as fine-particle ICS in controlling asthma symptoms $[6,7]$. Some patients typically excluded in these studies (see above) might differentially benefit from extrafine-particle ICS [4]. For these reasons, real-life database comparative effectiveness studies may usefully complement traditional RCTs to evaluate whether different properties of extrafine-particle and fine-particle ICS result in different effects in broader clinical populations.

Relevant real-life database studies in the UK and US show matched asthma patients prescribed extrafineparticle hydrofluoroalkane beclomethasone dipropionate (EF-HFA-BDP) for the first time had equivalent or better odds of achieving asthma control over 1 year than patients initiating fluticasone, despite being prescribed lower doses [8-10]. In contrast to findings from some RCTs $[6,7]$, these studies suggest that extrafine-particle ICS offers further clinical benefits in asthma control compared with fine-particle ICS therapy in real-life populations. To determine whether this finding can be generalized in other settings with different healthcare systems and possibly different prescribing habits, this real-life database study compared the effectiveness of initiating ICS therapy in patients with asthma in the Netherlands prescribed extrafine-particle ciclesonide or EF-HFA-BDP (Qvar ${ }^{\circledR}$ ) versus fine-particle ICS fluticasone propionate or non-extrafine-particle-beclomethasone dipropionate (Non-EF-BDP). In line with previous real-life research [8-10], it was hypothesized that EF-ICS may be at least as effective as fine-particle ICS therapy.

\section{Methods}

\section{Study design and data source}

This was a matched cohort, database study, consisting of a baseline and outcome period. The baseline period served for patient characterization and confounder definition and was 1 year before the initiation date of ICS therapy. The outcome period was 1 year following the initiation date for evaluating the effectiveness of ICS therapy. The initiation date was the date when patients received their first prescription of extrafine-particle ICS (either ciclesonide or EF-HFA-BDP), or fine-particle ICS (either fluticasone or Non-EF-BDP). The study period was from January 1998 to December 2012.

An independent steering committee was involved in a priori development of study design, review of analyses and interpretation of results [11]. The study was registered with the European Network of Centres for Pharmacoepidemiology and Pharmacovigilance (ENCePP, study no. 8391), and conducted in accordance with the ENCePP Code of Conduct. The study design is summarized in Additional file 1: Figure S1.

Anonymous data to inform the study objectives were obtained from the PHARMO Database Network provided by the PHARMO Institute for Drug Outcomes Research (Utrecht, the Netherlands). The PHARMO Database Network comprises, among other databases, linked Outpatient Pharmacy, General Practice (GP), Hospitalization and Clinical Laboratory Register databases, and accounts for almost 3 million patients', representative of $20 \%$ of the Dutch population. The Outpatient Pharmacy database includes the dispensing records of more than 200 community pharmacies and is linked to hospital discharge records. Data from the PHARMO Institute were provided in accordance with Dutch privacy laws.

\section{Study population}

The study population included adult patients aged 12-60 years with evidence of asthma, defined as $\geq 2$ prescriptions for asthma therapy at any time in their recorded history, a General Practice-recorded diagnosis for asthma (International Classification of Primary Care [ICPC] code R96), when available, and receiving continuous ICS therapy following the initiation date, defined as $\geq 2$ ICS prescriptions during the outcome period in addition to the first prescription. Patients were excluded from the study if, at any time, they had been diagnosed with any chronic respiratory diseases other than asthma. Patients prescribed long-acting muscarinic antagonists at baseline were not excluded.

\section{Outcomes}

The rate of severe asthma exacerbations in the year following ICS therapy initiation, one of three co-primary outcomes, was defined based on the American Thoracic 
Society/European Respiratory Society (ATS/ERS) Task Force definition to include asthma-related hospital admissions or prescription for acute courses of oral corticosteroids. Asthma-related admissions were defined as any hospital entry for asthma or any lower respiratory reason (including lower respiratory tract infections). Acute oral corticosteroid use associated with asthma exacerbation treatment was defined as all courses that were not maintenance therapy, and/or all courses where dosing instructions suggested exacerbation treatment. Emergency department data were not included in the exacerbation definition as these data were not available in the database. Two further co-primary outcomes included risk-domain asthma control, defined as the absence of asthma-related hospital admissions and prescription for acute courses of oral corticosteroids; and overall asthma control, defined as achieved risk-domain asthma control and average daily dose of $\leq 200 \mu \mathrm{g}$ salbutamol or $\leq 500 \mu \mathrm{g}$ terbutaline.

Secondary outcomes were (1) treatment stability during the outcome period, defined as achieving risk-domain asthma control and no change in therapy; and (2) average daily dose of short-acting $\beta 2$ agonists (SABA), defined as number of inhalers $\frac{\times \text { Doses per inhaler }}{365} \times$ strength, where strength is inhaler dose $(\mu \mathrm{g})$. Change in therapy was defined as an ICS prescribed dose increase of $\geq 50 \%$ or addition of new asthma therapy, including leukotriene receptor antagonists (LTRA), theophylline, or long-acting $\beta$ agonists (LABA).

Finally, two exploratory outcomes were (1) prevalence of oral candidiasis, based on the number and percentage of patients who either received a diagnosis of oral candidiasis in their hospital records, or the number and percentage of topical oral prescriptions for antifungals; and (2) hospitalization rate, defined as the number of any recorded hospital entry for asthma and/or any (asthma-related) lower respiratory reason in the year following the initiation date. All outcomes have been used in prior studies by the research group and have been described previously [8-10].

\section{Statistical analysis}

The database extraction and statistical analysis plan were written before any analyses were conducted [12]. All analysis was carried out using IBM SPSS Statistics version 22, Microsoft Office EXCEL 2007, and SAS version 9.3. Complete details on statistical analysis methods are in the Additional file 1: Figure S1.

Patients were exact-matched (1:1) based on key baseline demographic and clinical characteristics to ensure comparison of similar patients and to reduce potential confounders [13]. Matching criteria were informed from baseline differences between treatment cohorts as evidenced by exploratory analysis ( $t$-test/chi square test, $p<0.05$ ), from expert clinical advice, and from previous research experience, and included the following: sex, age, exact year of ICS therapy initiation, severe exacerbations, LABA and LTRA prescriptions, and SABA daily dose. Patients were matched sequentially on each of the selected matching criteria. Patients who did not match were excluded.

The rate of severe exacerbations in the outcome period was compared between matched treatment cohorts using a conditional Poisson regression model. The odds of achieving risk-domain asthma control and overall asthma control, and changing asthma therapy, were compared between matched treatment cohorts using a conditional binary logistic regression model. Ordinal conditional logistic regression modeling was used to analyze prescriptions of higher doses of SABA (categorized doses). The models used empirical standard errors (for more conservative confidence interval $[\mathrm{CI}]$ estimations) and adjustments were made for any residual non-collinear baseline confounders (Pearson and Spearman correlation coefficients, $r>0.3$ ) and for those variables predictive of the results through full multivariable analysis (multivariable model, $p \leq 0.05$ ). The results are expressed as adjusted rate ratios and odds ratios and $95 \%$ confidence intervals. Number of prescriptions of antifungal medications to treat oral candidiasis, prevalence of hospitalizations, and initial ICS doses were compared between the two matched cohorts through conditional logistic regression $(p<0.05)$.

\section{Results}

\section{Patient characteristics and demographics}

Following 1:1 matching, 1399 patients were selected in each of the extrafine-particle ICS and fine-particle ICS cohorts (Fig. 1).

Within the extrafine-particle cohort, 712 patients (51\%) were prescribed ciclesonide, 687 (49 \%) EF-HFA-BDP. Median (interquartile range [IQR]) age for patients in each of the matched cohorts was 43 years. Median (IQR) ICS doses $(\mu \mathrm{g})$ were $160(160-320)$ for the extrafine-particle cohort versus $500(250-500)$ for the fine-particle cohort $(p<0.001)$ (fluticasone-equivalents) (Fig. 2).

The two study cohorts were similar for the study asthma control measures and prescriptions for SABA (Table 1). However, at baseline, patients in the extrafineparticle cohort received more diagnoses for rhinitis and gastro-oesophageal reflux disease, more prescriptions for acute oral corticosteroids, topical corticosteroid therapy, and had a higher prevalence of asthma-related hospital admissions, than patients in the fine-particle cohort (Table 1). For these specific variables, data were comparable within the extrafine-particle cohort for patients prescribed ciclesonide and EF-HFA-BDP (Table 1). 


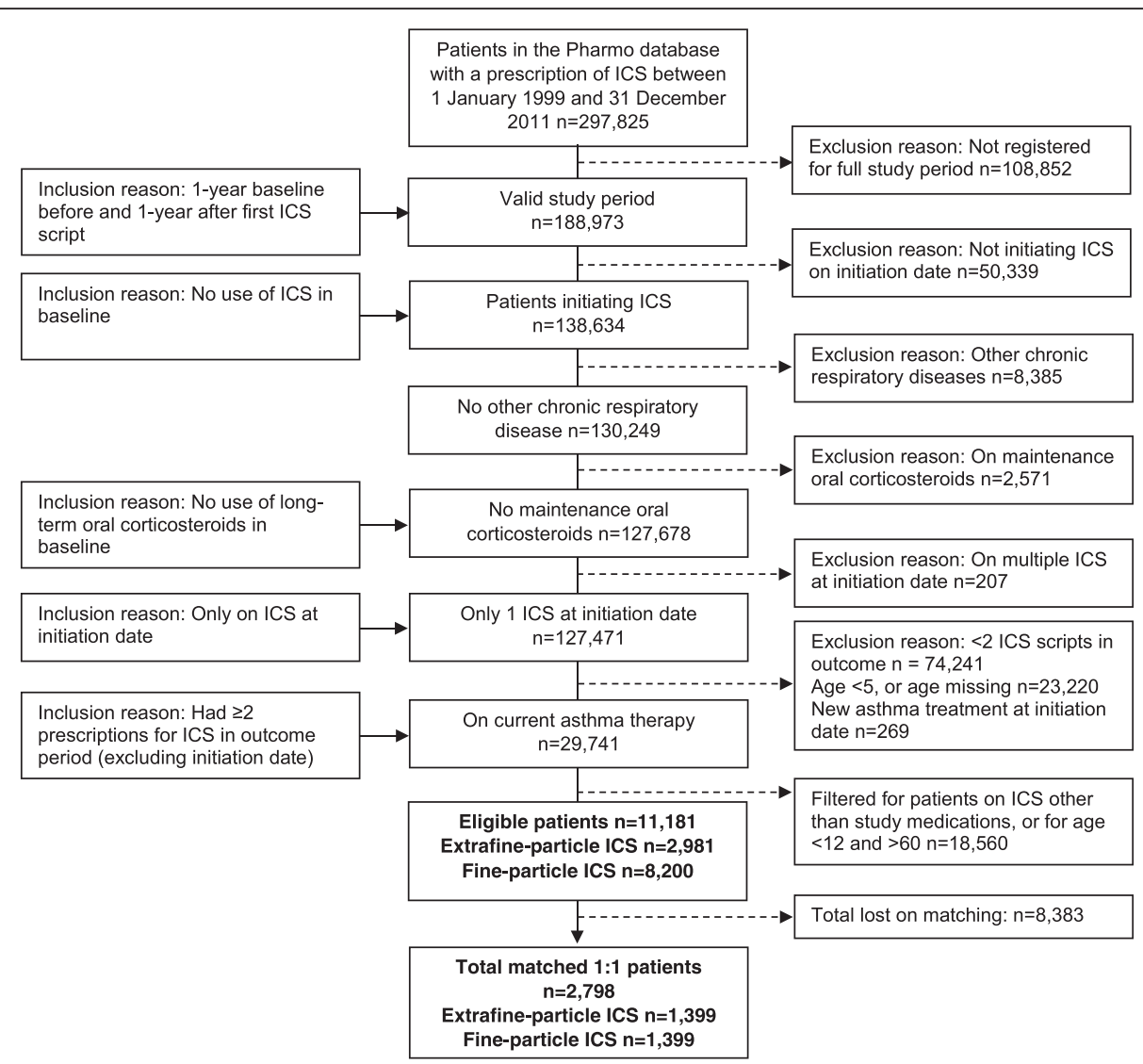

Fig. 1 Patient flow chart showing selection of patients in the PHARMO Database Network Patients in the two study cohorts were matched on clinically and demographically significant characteristics. PHARMO: PHARMO Database Network provided by the PHARMO Institute for Drug Outcomes Research (Utrecht, the Netherlands). ICS: inhaled corticosteroid; initiation date: the date when patients received their first prescription of extrafine-particle ICS (ciclesonide or EF-HFA-BDP), or fine-particle ICS (fluticasone or Non-EF-BDP)

\section{Outcomes}

Patients who received extrafine-particle ICS had $40 \%$ lower rates of severe exacerbations in the year following the initiation date than those receiving fine-particle ICS therapy, following adjustment for residual confounders (adjusted rate ratio [95 \% CI], 0.59 [0.47-0.73]) (Fig. 3). In addition, patients in the extrafine-particle cohort had greater odds of achieving risk-domain asthma control, overall asthma control, and treatment stability, and had lower odds of being in a higher dose category of

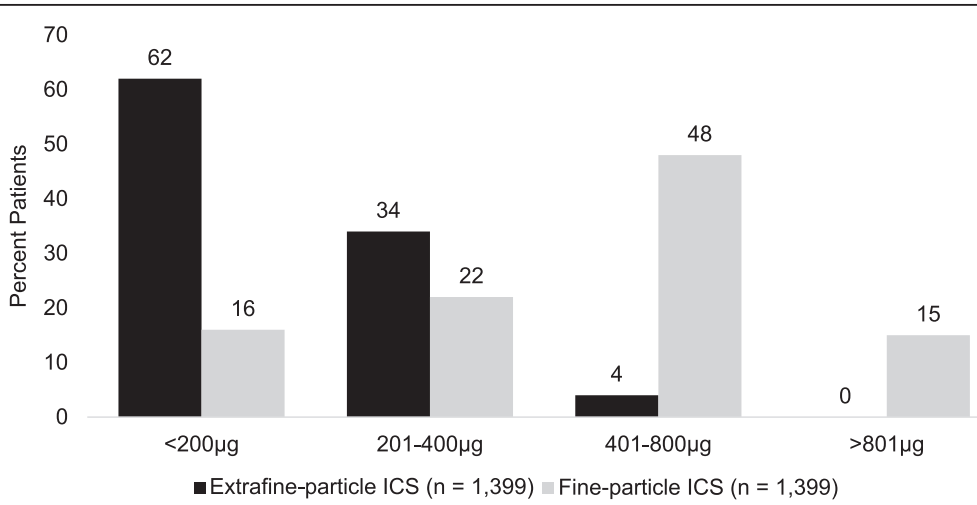

Fig. 2 Dose of inhaled corticosteroids prescribed on the initiation date* Reported doses are the actual dose for extrafine-particle ICS (ciclesonide or EF-HFA-BDP) and the fluticasone-equivalent dose for fine-particle ICS (fluticasone and Non-EF-BDP). * Initiation date: the date when patients received their first prescription of extrafine-particle ICS (ciclesonide or EF-HFA-BDP), or fine-particle ICS (fluticasone or Non-EF-BDP) 
Table 1 Baseline demographic and clinical characteristics for 1:1 matched patients

\begin{tabular}{|c|c|c|c|c|}
\hline \multicolumn{2}{|l|}{ Baseline Characteristic } & $\begin{array}{l}\text { Extrafine-particle } \\
\operatorname{ICS}^{a}(n=1399)\end{array}$ & $\begin{array}{l}\text { Fine-particle } I C S^{b} \\
(n=1399)\end{array}$ & $p$-value \\
\hline \multicolumn{2}{|l|}{ Sex, male, $n(\%)^{c}$} & $471(34)$ & $471(34)$ & $\mathrm{N} / \mathrm{A}$ \\
\hline \multicolumn{2}{|l|}{ Age, median $(\mathrm{QQR})^{\mathrm{c}}$} & $43(32-53)$ & $43(32-52)$ & 0.091 \\
\hline \multicolumn{2}{|l|}{ Year of initiation date, median $(\mathrm{IQR})^{\mathrm{C}}$} & $2008(2006-2009)$ & $2008(2006-2009)$ & N/A \\
\hline \multicolumn{5}{|l|}{ ICS dose prescribed on initiation date, n (\%) } \\
\hline \multicolumn{2}{|l|}{$<200 \mu \mathrm{g} / \mathrm{d}, \mathrm{n}(\%)$} & $866(62)$ & $218(16)$ & $<0.001$ \\
\hline \multicolumn{2}{|l|}{$201-400 \mu \mathrm{g} / \mathrm{d}, \mathrm{n}(\%)$} & $477(34)$ & $301(22)$ & \\
\hline \multicolumn{2}{|l|}{$401-800 \mu \mathrm{g} / \mathrm{d}, \mathrm{n}(\%)$} & $56(4)$ & $677(48)$ & \\
\hline \multicolumn{2}{|l|}{$801 \mu \mathrm{g} / \mathrm{d}, \mathrm{n}(\%)$} & $0(0)$ & $203(15)$ & \\
\hline \multicolumn{2}{|l|}{ ICS dose on initiation date, median (IQR) } & $160(160-320)$ & $500(250-500)$ & $<0.001$ \\
\hline \multicolumn{5}{|c|}{ Average ICS daily dose ( $\mu \mathrm{g} / \mathrm{d}$, categorized EF-HFA-BDP/ciclesonide equivalent dose ${ }^{d}$ ) } \\
\hline \multicolumn{2}{|l|}{$0-200 \mu \mathrm{g} / \mathrm{d}, \mathrm{n}(\%)$} & $866(62)$ & $218(16)$ & $<0.001$ \\
\hline \multicolumn{2}{|l|}{$201-400 \mu \mathrm{g} / \mathrm{d}, \mathrm{n}(\%)$} & $477(34)$ & $301(22)$ & \\
\hline \multicolumn{2}{|l|}{$401-800 \mu \mathrm{g} / \mathrm{d}, \mathrm{n}(\%)$} & $56(4)$ & $677(48)$ & \\
\hline \multicolumn{2}{|l|}{$801 \mu \mathrm{g} / \mathrm{d}+, \mathrm{n}(\%)$} & $0(0)$ & $203(15)$ & \\
\hline \multicolumn{5}{|l|}{ Recorded comorbidity, n (\%) } \\
\hline \multicolumn{2}{|l|}{ Rhinitis diagnosis, n (\%) } & $623(45)$ & $536(38)$ & $<0.001$ \\
\hline \multicolumn{2}{|l|}{ GERD diagnosis, n (\%) } & $513(37)$ & $437(31)$ & 0.001 \\
\hline \multicolumn{2}{|l|}{ Paracetamol script, n (\%) } & $28(2)$ & $47(3)$ & 0.020 \\
\hline \multicolumn{2}{|l|}{ NSAID script, n (\%) } & $219(16)$ & $215(15)$ & 0.834 \\
\hline \multicolumn{2}{|l|}{ Topical corticosteroid treatment, n (\%) } & $433(31)$ & $374(27)$ & 0.014 \\
\hline \multicolumn{2}{|l|}{ Oral candidiasis diagnosis, n (\%) } & $14(1)$ & $11(1)$ & 0.533 \\
\hline \multirow[t]{2}{*}{ Rhinitis diagnosis, n (\%) } & Ciclesonide & $18(1)$ & N/A & N/A \\
\hline & EF-HFA-BDP & $21(1)$ & & \\
\hline \multirow[t]{2}{*}{ GERD diagnosis, n (\%) } & Ciclesonide & $0(0)$ & N/A & N/A \\
\hline & EF-HFA-BDP & $1(1)$ & & \\
\hline \multirow[t]{2}{*}{ Topical corticosteroid treatment, n (\%) } & Ciclesonide & $207(15)$ & N/A & $\mathrm{N} / \mathrm{A}$ \\
\hline & EF-HFA-BDP & $226(16)$ & & \\
\hline Respiratory Medications & & & & \\
\hline Acute oral corticosteroid prescriptions, <1, n (\%) & & $101(7)$ & $120(9)$ & 0.001 \\
\hline Acute oral corticosteroid prescriptions, <1, n (\%) & Ciclesonide & $47(3)$ & N/A & N/A \\
\hline & EF-HFA-BDP & $54(4)$ & & \\
\hline SABA daily dose, $n(\%)^{c}$ & & & & \\
\hline O $\mu \mathrm{g} / \mathrm{d}, \mathrm{n}(\%)$ & & $953(68)$ & $953(68)$ & N/A \\
\hline $200 \mu \mathrm{g} / \mathrm{d}, \mathrm{n}(\%)$ & & $57(4)$ & $57(4)$ & \\
\hline $201 \mu \mathrm{g} / \mathrm{d}, \mathrm{n}(\%)$ & & $40(3)$ & $40(3)$ & \\
\hline SABA prescriptions, n (\%) & & & & \\
\hline $0, n(\%)^{c}$ & & $953(68)$ & $953(68)$ & 0.251 \\
\hline 1, n (\%) & & $281(20)$ & $267(19)$ & \\
\hline $2+, \mathrm{n}(\%)$ & & $165(12)$ & $179(13)$ & \\
\hline Prior therapy & & & & \\
\hline LABA, n $(\%)^{c}$ & & $21(2)$ & $21(2)$ & $\mathrm{N} / \mathrm{A}$ \\
\hline LTRA, n (\%) ${ }^{c}$ & & $1(0)$ & $1(0)$ & N/A \\
\hline
\end{tabular}


Table 1 Baseline demographic and clinical characteristics for 1:1 matched patients (Continued)

\begin{tabular}{|c|c|c|c|c|}
\hline \multicolumn{5}{|l|}{ Asthma control } \\
\hline Risk-domain asthma control, $\mathrm{n}(\%)^{c}$ & & $1275(91)$ & $1275(91)$ & N/A \\
\hline Overall asthma control, $\mathrm{n}(\%)^{\mathrm{c}}$ & & $1241(89)$ & $1241(89)$ & N/A \\
\hline \multicolumn{5}{|l|}{ Severe exacerbations, $n(\%)^{c}$} \\
\hline 0 & & $1275(91)$ & $1275(91)$ & N/A \\
\hline 1 & & $109(8)$ & $109(8)$ & \\
\hline $2+$ & & $15(1)$ & $15(1)$ & \\
\hline Asthma-related hospital admissions, n (\%) & & $23(2)$ & $4(0)$ & 0.001 \\
\hline \multirow[t]{2}{*}{ Asthma-related hospital admissions, n (\%) } & Ciclesonide & $11(1)$ & N/A & N/A \\
\hline & EF-HFA-BDP & $12(1)$ & & \\
\hline
\end{tabular}

GERD, gastro-oesophageal reflux disease; ICS, inhaled corticosteroid; $L A B A$, long-acting $\beta 2$-agonist; $L T R A$, leukotriene receptor antagonist; $N / A$ : not applicable; SABA, short-acting $\beta 2$-agonist; initiation date: the date when patients received their first prescription of extrafine-particle ICS (ciclesonide or EF-HFA-BDP), or fine-particle ICS (fluticasone or Non-EF-BDP)

${ }^{a}$ Extrafine-particle ICS: ciclesonide; hydrofluoroalkane beclomethasone dipropionate (EF-HFA-BDP)

${ }^{b}$ Fine-particle ICS: non-extrafine-particle beclomethasone dipropionate (Non-EF-BDP); fluticasone propionate

cMatching variable

${ }^{\mathrm{d}}$ Average ICS daily dose calculated as [(number of inhalers $*$ doses per inhaler / 365) $* \mu \mathrm{g}$ strength]

SABA (adjusted rate ratio $[95 \% \mathrm{CI}], 0.50[0.44-0.57]$ )

(Fig. 3, Table 2).

During the outcome year, patients in the extrafineparticle cohort received fewer prescriptions for other respiratory drugs (oral corticosteroids, SABA and LABA) compared with the fine-particle cohort, although more patients in the extrafine-particle cohort were prescribed LTRAs during the outcome period than the fine-particle cohort (6\% and $3 \%$ in extrafine-particle versus fine- particle ICS cohorts, respectively; $\mathrm{p}=0.001$ ) (Fig. 3, Table 2). Prescriptions of medications for treating oral candidiasis in the outcome period were not significantly different between the two study cohorts. Although patients in the extrafine-particle cohort had significantly higher asthma-related hospital admissions in baseline (Table 1), in the year following the date of first prescription of ICS therapy, this difference was not evident (Table 2).

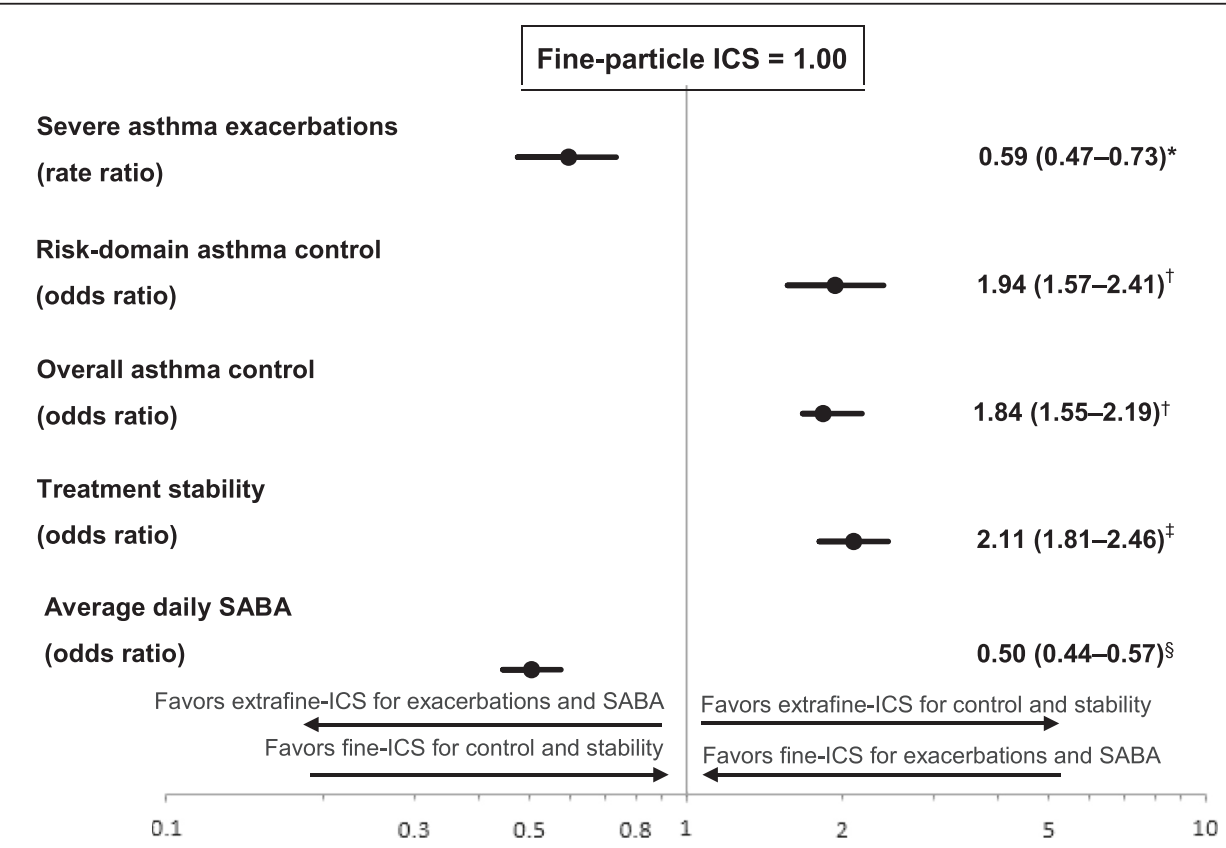

Fig. 3 Adjusted rate and odds ratios (95\% Cl) for co-primary and secondary outcomes. ICS: inhaled corticosteroid; SABA, short-acting $\beta 2$-agonist. *Adjusted for baseline exacerbations (ATS/ERS Definition, categorized), evidence of GERD and baseline asthma prescriptions (categorized); ${ }^{+}$Adjusted for baseline Risk-domain asthma control, evidence of GERD and asthma diagnosis; ${ }^{\ddagger}$ Adjusted for evidence of rhinitis, evidence of GERD and baseline Risk-domain asthma control; ${ }^{\S}$ Adjusted for baseline SABA daily dose $(\mu \mathrm{g})$ 
Table 2 Outcome measures for matched groups 1 year following date of first ICS prescription

\begin{tabular}{|c|c|c|c|}
\hline Outcome & $\begin{array}{l}\text { Extrafine-particle ICS } \\
(n=1399)\end{array}$ & $\begin{array}{l}\text { Fine-particle ICS } \\
(n=1399)\end{array}$ & $p$-value \\
\hline \multicolumn{4}{|l|}{ Asthma control } \\
\hline Risk-domain asthma control, n (\%) & $1236(88)$ & $1129(81)$ & $<0.001$ \\
\hline Overall asthma control, n (\%) & $1091(78)$ & $934(67)$ & $<0.001$ \\
\hline \multicolumn{4}{|l|}{ Severe exacerbations, n (\%) } \\
\hline $0, \mathrm{n}(\%)$ & $1236(88)$ & $1129(81)$ & \multirow[t]{3}{*}{$<0.001$} \\
\hline $1, \mathrm{n}(\%)$ & $108(8)$ & $184(13)$ & \\
\hline $2+, n(\%)$ & $55(4)$ & $86(6)$ & \\
\hline Treatment stability, n (\%) & $809(58)$ & $560(40)$ & $<0.001$ \\
\hline \multicolumn{4}{|l|}{ Prescribed average daily dose of SABA, $\mu \mathrm{g} / \mathrm{d}, \mathrm{n}(\%)$} \\
\hline $0 \mu \mathrm{g} / \mathrm{d}, \mathrm{n}(\%)$ & $686(49)$ & $461(33)$ & \multirow[t]{3}{*}{$<0.001$} \\
\hline $1-100 \mu \mathrm{g} / \mathrm{d}, \mathrm{n}(\%)$ & $326(23)$ & $343(25)$ & \\
\hline $101 \mu \mathrm{g} / \mathrm{d}+, \mathrm{n}(\%)$ & $387(28)$ & $595(43)$ & \\
\hline $1+$ prescriptions of antifungal for candidiasis, $\mathrm{n}(\%)$ & $48(3)$ & $45(3)$ & 0.753 \\
\hline 1+ asthma-related hospital admissions, n (\%) & $19(1)$ & $16(1)$ & 0.613 \\
\hline \multicolumn{4}{|l|}{ Respiratory medications in outcome period } \\
\hline \multicolumn{4}{|l|}{ Courses of acute oral corticosteroids, n (\%) } \\
\hline $0, \mathrm{n}(\%)$ & $1246(89)$ & $1135(81)$ & \multirow[t]{3}{*}{$<0.001$} \\
\hline $1, \mathrm{n}(\%)$ & $102(7)$ & $182(13)$ & \\
\hline $2+, \mathrm{n}(\%)$ & $51(5)$ & $82(6)$ & \\
\hline \multicolumn{4}{|l|}{ SABA prescriptions, $n(\%)$} \\
\hline $0, \mathrm{n}(\%)$ & $686(49)$ & $461(33)$ & \multirow[t]{4}{*}{$<0.001$} \\
\hline $1, \mathrm{n}(\%)$ & $245(18)$ & $235(17)$ & \\
\hline $2, \mathrm{n}(\%)$ & $180(13)$ & $218(16)$ & \\
\hline $3+, n(\%)$ & $288(21)$ & $485(35)$ & \\
\hline \multicolumn{4}{|l|}{ ICS prescriptions (including initial prescription) } \\
\hline $2, n(\%)$ & $0(0)$ & $0(0)$ & \multirow[t]{3}{*}{0.022} \\
\hline $3, \mathrm{n}(\%)$ & $462(33)$ & $520(37)$ & \\
\hline $4+, n(\%)$ & $937(67)$ & $879(63)$ & \\
\hline \multicolumn{4}{|l|}{ Average ICS daily dose ( $\mu \mathrm{g} / \mathrm{d}$, fluticasone-equivalents ${ }^{\complement}$ ) } \\
\hline $0-150 \mu \mathrm{g} / \mathrm{d}, \mathrm{n}(\%)$ & $460(33)$ & $263(19)$ & \multirow[t]{4}{*}{$<0.001$} \\
\hline $151-250 \mu \mathrm{g} / \mathrm{d}, \mathrm{n}(\%)$ & $484(35)$ & $416(30)$ & \\
\hline $251-450 \mu \mathrm{g} / \mathrm{d}, \mathrm{n}(\%)$ & $358(26)$ & $401(29)$ & \\
\hline $451 \mu \mathrm{g} / \mathrm{d}+\mathrm{n}(\%)$ & $97(7)$ & $319(23)$ & \\
\hline $\begin{array}{l}\text { Average ICS daily dose }(\mu \mathrm{g} / \mathrm{d} \text {, EF-HFA-BDP/ } \\
\left.\text { ciclesonide equivalent dose }^{\mathrm{d}}\right) \text {, median (IQR) }\end{array}$ & $185(132-290)$ & $272(178-410)$ & $<0.001$ \\
\hline \multicolumn{4}{|l|}{$\begin{array}{l}\text { Average ICS daily dose }(\mu \mathrm{g} / \mathrm{d} \text {, EF-HFA-BDP/ } \\
\left.\text { ciclesonide equivalent dose }{ }^{\prime}\right)\end{array}$} \\
\hline $0-150 \mu \mathrm{g} / \mathrm{d}, \mathrm{n}(\%)$ & $460(33)$ & $263(19)$ & \multirow[t]{4}{*}{$<0.001$} \\
\hline $151-250 \mu \mathrm{g} / \mathrm{d}, \mathrm{n}(\%)$ & $484(35)$ & $416(30)$ & \\
\hline $251-450 \mu \mathrm{g} / \mathrm{d}, \mathrm{n}(\%)$ & $358(26)$ & $401(29)$ & \\
\hline $451 \mu \mathrm{g} / \mathrm{d}+, \mathrm{n}(\%)$ & $97(7)$ & $319(23)$ & \\
\hline LABA prescriptions in outcome period & $770(55)$ & $875(63)$ & $<0.001$ \\
\hline LTRA prescriptions in outcome period & $83(6)$ & $47(3)$ & 0.001 \\
\hline
\end{tabular}

GERD gastro-oesophageal reflux disease, ICS inhaled corticosteroid, LABA long-acting $\beta 2$-agonist, LTRA leukotriene receptor antagonist, N/A not applicable, SABA short-acting $\beta 2$-agonist

${ }^{a}$ Extrafine-particle ICS: ciclesonide (Alvesco ${ }^{\circledast}$ ); hydrofluoroalkane beclomethasone dipropionate (EF-HFA-BDP [Qvar $\left.{ }^{\circledast}\right]$ )

${ }^{b}$ Fine-particle ICS: non-extrafine-particle beclomethasone dipropionate (Non-EF-BDP); fluticasone propionate (FP)

${ }^{c}$ Fluticasone-equivalent dose for fine-particle ICS (FP and Non-EF-BDP) delivered via a pressurized-metered dose inhaler

${ }^{d}$ EF-HFA-BDP/ciclesonide equivalent dose for extrafine-particle ICS delivered via a pressurized-metered dose inhaler 


\section{Discussion}

In this real-life population-based study, matched patients initiating extrafine-particle ICS had significantly lower rates of severe exacerbations and significantly higher odds of achieving asthma control and treatment stability than those prescribed fine-particle ICS. Notably, the prescribed doses of extrafine-particle ICS were lower than fine-particle ICS at the initiation date (median dose, 160 vs. $500 \mu \mathrm{g}$ per day, fluticasone-equivalents). In addition, patients prescribed extrafine-particle ICS showed lower odds of being prescribed higher doses of short-acting $\beta 2$ agonists. Outcome data showed no significant differences between the study cohorts for medications prescribed for treating oral candidiasis, and for asthma-related hospital admissions. These findings suggest a significant improvement in asthma control for patients prescribed extrafine-particle ICS with unstable asthma. Such patients would typically be excluded from traditional RCTs.

Possible mechanisms to account for the superior effectiveness of extrafine-particle ICS observed in this study include improved airway drug deposition and distribution, including distally in the small airways, and improved inhaler device tolerance. These are each discussed in turn below.

Aerosol particle size now appears therapeutically important for controlling asthma symptoms [14]. Evidence indicates that fine-particle ICS with a MMAD of $<5 \mu \mathrm{m}$, but $\geq 2 \mu \mathrm{m}$, show lower total lung distribution and deposition than extrafine-particles with a MMAD $<2 \mu \mathrm{m}$, which can deposit more in the small airways $[1,2,15]$. More effective control of small airway inflammation might contribute to improved asthma control [15].

Poor device technique by patients is a common problem observed by clinicians. Asthma control worsens as the number of mistakes in device technique increases [16]. The combination of fine-particle ICS with poor device technique can lead to some of the ICS settling in the oropharynx, resulting in side-effects such as oropharyngeal candidiasis. However, lung deposition of EF-HFA-BDP remains adequate even in patients with poor inhaler device technique [1]. This may be due to pMDI design incorporating hydrofluoroalkane propellant that produces a softer, warmer and longer duration spray. This particular method of delivery has proven more tolerant of poor inhaler technique than some metered dose inhalers emitting fine-particle ICS [1]. Tolerance to inhalation errors may in turn promote improved adherence to therapy [14].

This study has several strengths. To ensure all potentially relevant variables for characterizing patients were included, and that the key outcomes of interest could be evaluated, both the statistical analysis plan, study population and outcomes were conceived prior to any analyses [11]. Imitating traditional RCT design, the study included an initiation date marking therapy initiation.
The study inclusion and exclusion criteria were selected to minimize potential confounding factors such as other asthma therapies, and to identify patients from a large clinical population receiving initial ICS therapy. Patient matching was used to adjust for demographic and clinical differences between the study cohorts. An advantage of 1-year baseline and outcome periods is that this timeperiod allows for natural seasonal changes in respiratory disease, and for recording infrequent clinical events such as exacerbations and asthma-related hospitalizations [11]. The results confirm both the study hypothesis and similar findings observed in real-life asthma control studies with patients in the UK and US [8-10]. This seems to suggest that the present findings can be extrapolated to healthcare systems and prescribing patterns in different countries. Collectively, these studies provide balance to findings reported in some RCTs that extrafine-particle ICS is only equally effective as fineparticle ICS in achieving asthma control [3, 4].

However, as with all real-life comparative database studies, this study has its limitations as well. Limitations could derive from using the PHARMO Database Network, including disease misclassification biases from the almost exclusive use of "asthma prescriptions" to individuate patients with asthma, and the impossibility to adjust for all potential confounders, such as potential confounding by severity for factors indiscernible from patient records or patient-reported outcomes. Asthma diagnosis data, defined as a General Practice recorded diagnosis for asthma (ICPC code R96), were available in the General Practice database for only $12.2 \%$ of the study population. In addition, the results of this study only apply to healthier patients who survive at least 1 year following prescription date. Therefore, this study cannot exclude potential survivor bias [11, 13, 17].

Although specific to respiratory disease, this study could not accurately assess lung function, or symptom control. However, short-acting $\beta 2$ agonist prescriptions were included in the study as a substitute for asthma symptoms in the 'overall asthma control' measure, because short-acting $\beta 2$ agonist use reflects symptom control [18]. The control cutoff point of a mean shortacting $\beta 2$ agonist use of $\geq 2$ puffs per day corresponds to the level 2 category ( 2 of 4 , with 1 being the best controlled) of the validated approach of Schatz et al. [18] for short-acting $\beta 2$ agonist canister dispensing to assess asthma symptom control. During the study period, 1399/2981 (47 \%) eligible patients initiating extrafineparticle ICS therapy were matched, and 1399/8200 (17\%) of those initiating fine-particle ICS were matched, possibly indicating that patients in neither cohort were representative of those who initiate extrafine-particle or fine-particle ICS therapy in the Netherlands. Finally, 
although smoking status was not reported in the study due to poor availability within the PHARMO Database Network, possible COPD patients were included (e.g., patients prescribed long-acting muscarinic agents in addition to ICS), but constituted only $1.8 \%$ of the total study population.

\section{Conclusions}

This study in a real-life population shows that extrafineparticle ICS are associated with better asthma control and better odds of therapy stability than fine-particle ICS, at significantly lower prescribed doses, despite patients having otherwise similar baseline characteristics after exact matching. These results for a Dutch population confirm relevant real-life asthma studies conducted in the UK and US, and seem generalizable to countries with different healthcare systems and prescribing habits. The findings also provide balance to findings reported in some randomized asthma RCTs. The study applied reliable prescribing and medical information recorded in a well-maintained database for a diverse clinical population, a population including those at high risk of exacerbations, with comorbid rhinitis, smokers and non-smokers. Although patients prescribed extrafineparticle ICS showed signs of more severe asthma, they had better asthma control at lower ICS doses than patients prescribed fine-particle ICS. Additional comparative effectiveness studies are required to better understand the differential effects of extrafine-particle versus fine-particle ICS in real-life asthma patients, especially regarding possible subgroup specificities.

\section{Additional file}

Additional file 1: Figure S1. Study design. (DOCX $84 \mathrm{~kb}$ )

\section{Abbreviations \\ EF-HFA-BDP [Qvar ${ }^{\circledast}$ : extrafine-particle hydrofluoroalkane beclomethasone dipropionate; FP: fluticasone propionate; GERD: gastro- oesophageal reflux disease; ICS: inhaled corticosteroid; LABA: Iong- acting 32 -agonist; LTRA: leukotriene receptor antagonist; N/A: not applicable; Non-EF-BDP: non-extrafine-particle beclomethasone dipropi- onate; PHARMO: PHARMO Database Network provided by the PHARMO Institute for Drug Outcomes Research (Utrecht, the Netherlands); SABA: short-acting $\beta 2$-agonist.}

\section{Competing interests}

Thys van der Molen has received grants for research, travel, and reimbursement for presentations and advisory boards from AstraZeneca, GlaxoSmithKline, Almirall, Mundipharma, Boehringer Ingelheim, Chiesi, Teva Pharmaceuticals Europe B.V., and Novartis.

David B. Price has Board Membership with Aerocrine, Almirall, Amgen, AstraZeneca, Boehringer Ingelheim, Chiesi, Meda, Mundipharma, Napp, Novartis, and Teva Pharmaceuticals Europe B.V. Consultancy: Almirall, Amgen, AstraZeneca, Boehringer Ingelheim, Chiesi, GlaxoSmithKline, Meda, Mundipharma, Napp, Novartis, Pfizer, Teva Pharmaceuticals Europe B.V., and Zentiva. Grants/Grants Pending with UK National Health Service, British Lung Foundation, Aerocrine, AstraZeneca, Boehringer Ingelheim, Chiesi, Eli Lilly, GlaxoSmithKline, Meda, Merck, Mundipharma, Novartis, Orion, Pfizer, Respiratory Effectiveness Group, Takeda, Teva Pharmaceuticals Europe B.V., and Zentiva. Payments for lectures/speaking: Almirall, AstraZeneca,
Boehringer Ingelheim, Chiesi, Cipla, GlaxoSmithKline, Kyorin, Meda, Merck, Mundipharma, Novartis, Pfizer, SkyePharma, Takeda, and Teva Pharmaceuticals Europe B.V. Payment for manuscript preparation: Mundipharma and Teva Pharmaceuticals Europe B.V. Patents (planned, pending or issued) with AKL Ltd. Payment for the development of educational materials: GlaxoSmithKline, and Novartis. Stock/Stock options: Shares in AKL Ltd, which produces phytopharmaceuticals. Owns $80 \%$ of Research in Real Life Ltd and its subsidiary social enterprise Optimum Patient Care. Received Payment for travel/accommodations/meeting expenses from: Aerocrine, Boehringer Ingelheim, Mundipharma, Napp, Novartis, and Teva Pharmaceuticals Europe B.V. Funding for patient enrolment or completion of research: Almirral, Chiesi, Teva Pharmaceuticals Europe B.V., and Zentiva. Peer reviewer for grant committees: Medical Research Council (2014), Efficacy and Mechanism Evaluation program (2012), HTA (2014); and received unrestricted funding for investigator-initiated studies from Aerocrine, AKL Ltd, Almirall, Boehringer Ingelheim, Chiesi, Meda, Mundipharma, Napp, Novartis, Orion, Takeda, Teva Pharmaceuticals Europe B.V., and Zentiva.

Ron M.C. Herings and Jetty A. Overbeek are employees of the PHARMO Institute for Drug Outcomes Research. This independent research institute performs financially supported studies for government and related healthcare authorities and several pharmaceutical companies.

Victoria Thomas is an employee of Research in Real-Life (RiRL). Cristiana Miglio is a former employee of Research in Real-Life, Ltd (RiRL). RiRL conducted this study. RiRL has conducted paid research in respiratory disease on behalf of the following other organizations in the past 5 years: Aerocrine, AKL Ltd, Almirall, AstraZeneca, Boehringer Ingelheim, Chiesi, GlaxoSmithKline, Meda, Mundipharma, Napp, Novartis, Orion, Takeda, Teva Pharmaceuticals Europe B.V., and Zentiva, a Sanofi company.

Richard Dekhuijzen has received over the past 3 years' fees for speaking, education, participation in advisory boards or consulting, from: Almirall, AstraZeneca, Boehringer Ingelheim, Chiesi, Mundipharma, Novartis, Pfizer, Teva Pharmaceuticals Europe B.V., and Zambon. Research grants: AstraZeneca, Boehringer Ingelheim, and Novartis.

Richard J. Martin has conducted consultancy work and/or received travel support and/or honoraria for attendance at advisory boards for: AstraZeneca, Medlmmune, Merck, and Teva Pharmaceuticals Europe B.V. Received research grants: Medlmmune, and the NHLBI. Received royalties from UpToDate. The University of Groningen has received money for Dirkje S. Postma regarding an unrestricted educational grant for research from Astra Zeneca, and Chiesi. Travel to ERS and/or ATS has been partially funded by Astra Zeneca, Chiesi, GlaxoSmithKline, and Takeda. Fees for consultancies given to the University of Groningen from: Astra Zeneca, Boehringer Ingelheim, Chiesi, GlaxoSmithKline, Takeda and Teva Pharmaceuticals Europe B.V. Travel and lectures in China paid by Chiesi.

Nicolas Roche has received fees over the past 3 years for speaking, education, and participation in advisory boards or consulting from: 3M, Aerocrine, Almirall, AstraZeneca, Boehringer Ingelheim, Chiesi, Cipla, GlaxoSmithKline, MSDChibret, Mundipharma, Novartis, Pfizer, Sandoz, Sanofi, Stallergenes, Takeda, and Teva Pharmaceuticals Europe B.V. Research grants from: Boehringer Ingelheim, Novartis, and Pfizer.

Theresa Guilbert has received personal fees from the American Board of Pediatrics, GlaxoSmithKline, Merck, and Regeneron Pharmaceuticals, and Sanofi. Pediatric Pulmonary Subboard, grants and personal fees from Teva Pharmaceuticals Europe B.V. Grants: Abbott Laboratories, Array Biopharma, CDC, CF Foundation Therapeutics, CompleWare Corporation, DHHS, F. Hoffman-LaRoche, Forest Research Institute, KaloBios Pharmaceuticals, Medimmune, Mylan, NIH, Roche/Genetech, Roxane Laboratories, and UW-Madison Medical and Education Research Committee, and Vertex Pharmaceuticals. Royalties from UpToDate. Elliot Israel reports having received speaking fees over the past 3 years' fees from Merck. Fees for consulting from AstraZeneca and Merck. Non-financial participation in Advisory Boards or consulting for Novartis (as DSMB Member). Research grants from Genentech and NIH. Writing/editorial support for manuscript preparation from RiRL. Travel grant support from RiRL and Teva Pharmaceuticals Europe B.V. Specialty Pharmaceuticals, fees for expert testimony from: Campbell, Campbell, Edwards \& Conroy, Ficksman \& Conley, Fox Rothschild, LLP, and Ryan Deluca, LLP. Royalties from UpToDate. Deputy Editor for the American Journal of Respiratory and Critical Care Medicine.

Wim van Aalderen is a member of the Medical Advisory Board of AstraZeneca. Elizabeth $V$. Hillyer is a consultant to RiRL and has received payment for writing and editorial support to Merck. 
Simon van Rysewyk is an employee of the Observational \& Pragmatic Research Institute Pte Ltd, and has no competing interests to declare.

\section{Authors' contributions}

All authors were involved in the conception or design of the study; or the acquisition, analysis, or interpretation of study data; and, drafting the study or revising it; and, giving final approval for publication. The authors are responsible for all aspects of this study. DBP led the study design process, which was guided by Steering Committee discussions. The Steering Committee was composed of study authors representing the SASG: RJM, DSP, NR, WVA, RD, and TvdM. CM and VT were responsible for the data acquisition and analyses. SvR wrote the manuscript.

\section{Acknowledgements}

Gokul Gopalan (a Senior Global Medical Director [Respiratory], at Teva Pharmaceuticals, Frazer, PA, US, at the time of this study), assisted with study design.

\section{Funding}

Funds to acquire the dataset from the PHARMO Institute for Drug Outcomes Research (Utrecht, the Netherlands) were provided by RiRL. The study received institutional support from Teva Pharmaceuticals Europe B.V. Gokul Gopalan, a Senior Global Medical Director (Respiratory), at Teva Pharmaceuticals, Frazer, PA, US, at the time of this study, assisted with study design, but neither Teva Pharmaceuticals Europe B.V. nor Teva Pharmaceuticals, Frazer, PA, US, contributed, either in part or in whole, to the collection, analysis, or interpretation of study data, manuscript writing, or the decision to submit the manuscript for publication.

\section{Author details}

${ }^{1}$ Department of General Practice, University of Groningen, University Medical Center, Groningen, The Netherlands. 'Department Pulmonary Medicine and Tuberculosis, University of Groningen, University Medical Center Groningen, Groningen, The Netherlands. ${ }^{3}$ National Jewish Health and the University of Colorado, Denver, USA. ${ }^{4} \mathrm{PHARMO}$ Institute for Drug Outcomes Research, Utrecht, The Netherlands. ${ }^{5}$ Research in Real Life, Ltd, Cambridge, UK. ${ }^{6}$ Radboud University Medical Centre, Nijmegen, The Netherlands. ${ }^{7}$ Groupe Hospitalier Cochin, AP-HP and University Paris Descartes (EA2511), Paris, France. ${ }^{8}$ Cincinnati Children's Hospital and Medical Center, Cincinnati, USA. ${ }^{9}$ Brigham and Women's Hospital and Harvard Medical School, Boston, MA, USA. ${ }^{10}$ Emma's Children Hospital, Academic Medical Centre, University of Amsterdam, Amsterdam, The Netherlands. ${ }^{11}$ Observational \& Pragmatic Research Institute Pte, Ltd, Singapore, Singapore. ${ }^{12}$ Academic Primary Care, University of Aberdeen, Polwarth Building, Foresterhill, AB25 2ZD, Aberdeen, UK.

Received: 24 December 2015 Accepted: 27 April 2016

Published online: 17 May 2016

\section{References}

1. Herland K, Akelesen J, Skjønsberg OH, Bjermer L. How representative are clinical study patients with asthma or COPD for a larger 'real life' population of patients with obstructive lung disease? Respir Med. 2005;99:11-9.

2. Travers J, Marsh S, Williams M, Weatherall M, Caldwell B, Shirtcliffe P, et al. External validity of randomised controlled trials in asthma: to whom do the results of the trials apply? Thorax. 2007;62(3):219-23.

3. Price D, Chisholm A, van der Molen T, Roche N, Hillyer EV, Bousquet J. Reassessing the evidence hierarchy in asthma: evaluating comparative effectiveness. Curr Allergy Asthma Rep. 2011;11(6):526-38.

4. Leach CL, Davidson PJ, Hasselquist BE, Boudreau RJ. Influence of particle size and patient dosing technique on lung deposition of HFA-beclomethasone from a metered dose inhaler. J Aerosol Med. 2005;18(4):379-85.

5. Leach CL, Bethke TD, Boudreau RJ, Hasselquist BE, Drollmann A, Davidson P, et al. Two-dimensional and three-dimensional imaging show ciclesonide has high lung deposition and peripheral distribution: a nonrandomized study in healthy volunteers. J Aerosol Med. 2006;19:117-26.

6. Aubier M, Wettenger R, Gans SJ. Efficacy of HFA-beclomethasone dipropionate extra-fine aerosol (800 mg day-1) versus HFA-fluticasone propionate (1000 mg day-1) in patients with asthma. Respir Med. 2001;95:212-20.
7. Fairfax A, Hall I, Spelman R. A randomized, double-blind comparison of beclomethasone dipropionate extrafine aerosol and fluticasone propionate. Ann Allergy Asthma Immunol. 2001;86:575-82.

8. Price D, Martin RJ, Barnes N, Dorinsky P, Israel E, Roche N, et al. Prescribing practices and asthma control with hydrofluoroalkane-beclomethasone and fluticasone: a real-world observational study. J Allergy Clin Immunol. 2010;126(3):511-18.

9. Price D, Thomas M, Haughney J, Lewis RA, Burden A, von Ziegenweidt J, et al. Real-life comparison of beclometasone dipropionate as an extrafineor larger-particle formulation for asthma. Respir Med. 2013;107(7):987-1000.

10. Colice G, Martin RJ, Israel E, Roche N, Barnes N, Burden A, et al. Asthma outcomes and costs of therapy with extrafine beclomethasone and fluticasone. J Allergy Clin Immunol. 2013;132(1):45-54.

11. Roche NRH, Martin R, Brusselle G, Papi A, Thomas M, Postma D, et al. Respiratory effectiveness group: quality standards for real-world research. Focus on observational database studies of comparative effectiveness. Ann Am Thorac Soc. 2014;11 Suppl 2:S99-S104.

12. Price DHE, van der Molen T. Efficacy versus effectiveness trials: informing guidelines for asthma management. Curr Opin Allergy Clin Immunol. 2013:13:50-7.

13. Stuart EA. Matching methods for causal inference: a review and a look forward. Stat Sci. 2010:25:1-21.

14. Newman S, Salmon SA, Nave R, Drollmann A. High lung deposition of 99mTc-labeled ciclesonide administered via HFA-MDI to patients with asthma. Respir Med. 2006;100:375-84.

15. Martin RJ. Therapeutic significance of distal airway inflammation in asthma. J Allergy Clin Immunol. 2002;109(2 Suppl):S447-S60.

16. Giraud V, Roche N. Misuse of corticosteroid metered-dose inhaler is associated with decreased asthma stability. Eur Respir J. 2002;19(2):246-51.

17. MacMahon S, Collins R. Reliable assessment of the effects of treatment on mortality and major morbidity, II: observational studies. Lancet. 2001;357:455-62.

18. Schatz M, Zeiger RS, Vollmer WM, Mosen D, Apter AJ, Stibolt TB, et al. Validation of a beta-agonist long-term asthma control scale derived from computerized pharmacy data. J Allergy Clin Immunol. 2006;117:995-1000.

\section{Submit your next manuscript to BioMed Central and we will help you at every step:}

- We accept pre-submission inquiries

- Our selector tool helps you to find the most relevant journal

- We provide round the clock customer support

- Convenient online submission

- Thorough peer review

- Inclusion in PubMed and all major indexing services

- Maximum visibility for your research

Submit your manuscript at www.biomedcentral.com/submit
Biomed Central 